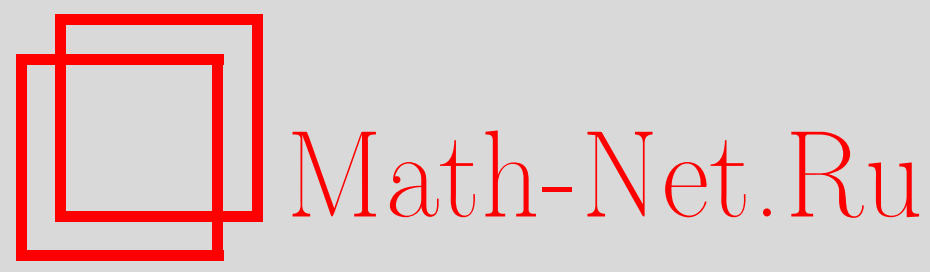

В. Г. Саргсян, Число разностей в группах простого порядка, Дискрет. матем., 2013, том 25, выпуск 1, 152-158

DOI: https://doi.org/10.4213/dm1228

Использование Общероссийского математического портала Math-Net.Ru подразумевает, что вы прочитали и согласны с пользовательским соглашением http://www . mathnet.ru/rus/agreement

Параметры загрузки:

IP: 54.196.121.252

26 апреля 2023 г., 06:12:38 


\title{
Число разностей \\ в группах простого порядка
}

\author{
() 2013 г. $\quad$ В. Г. Саргсян
}

Подмножество $A$ элементов группы $\mathbf{G}$ называется разностью, если существует такое подмножество $B \subseteq \mathbf{G}$, что $A=B-B$, где

$$
B-B=\{x-y \mid x, y \in B\} .
$$

Получена асимптотика логарифма числа разностей в группах простого порядка.

Работа выполнена при поддержке Российского фонда фундаментальных исследований, проект 13-01-00958-А.

\section{1. Введение}

Пусть $\mathbf{Z}_{p}$ - группа вычетов по простому модулю $p$, и $A \subseteq \mathbf{Z}_{p}$. Обозначим

$$
\begin{aligned}
A+A & =\left\{x_{1}+x_{2} \mid x_{1}, x_{2} \in A\right\}, \\
A-A & =\left\{x_{1}-x_{2} \mid x_{1}, x_{2} \in A\right\}, \\
-A & =\{-x \mid x \in A\} .
\end{aligned}
$$

Пусть $k \geqslant 0$ и $l \geqslant 0-$ целые числа, удовлетворяющие условию $k+l \geqslant 2$. Подмножество $A \subseteq \mathbf{Z}_{p}$ называется $(k, l)$-суммой, если существует подмножество $B \subseteq \mathbf{Z}_{p}$ такое, что

$$
A=\underbrace{B+\ldots+B}_{k}-\underbrace{B-\ldots-B}_{l} .
$$

В частности, $(1,1)$-сумму назовем разностью. Семейство $(k, l)$-сумм в $\mathbf{Z}_{p}$ обозначим через $\mathbf{S S}_{k, l}\left(\mathbf{Z}_{p}\right)$.

В 2004 г. Б. Грин и И. Ружа доказали следующее утверждение [1].

Теорема 1. Пусть р- простое число. Тогда справедливы оценки

$$
p^{2} 2^{p / 3} \ll\left|\mathbf{S S}_{2,0}\left(\mathbf{Z}_{p}\right)\right| \leqslant 2^{p / 3+\varkappa(p)},
$$

где $\varkappa(p) / p \rightarrow 0$ при $p \rightarrow \infty$, причем

$$
\varkappa(p) \ll p(\log \log p)^{2 / 3}(\log p)^{-1 / 9} ;
$$

здесь и далее логарифмы берутся по основанию два. 
В 2012 г. автором доказано следующее утверждение [2].

Теорема 2. Пусть $p$ - достаточно большое простое число, $k \geqslant 0$ u $l \geqslant 0$ - иелье числа, удовлетворяющие условию $k+l \geqslant 2$. Тогда выполняются неравенства

$$
C_{k, l} 2^{p /(2(k+l)-1)} \leqslant\left|\mathbf{S S}_{k, l}\left(\mathbf{Z}_{p}\right)\right| \leqslant 2^{p /(k+l+1)+o(p)},
$$

где $C_{k, l}-$ некоторая положительная константа.

Целью настоящей работы является улучшение оценок в теореме 2 на случай $k=1$ и $l=1$. Мы докажем следующее утверждение.

Теорема 3. Пусть р- простое число. Тогда справедливы соотношения

$$
p^{2} 2^{p / 3} \ll\left|\mathbf{S S}_{1,1}\left(\mathbf{Z}_{p}\right)\right| \leqslant 2^{p / 3+\varkappa(p)},
$$

где $\varkappa(p) / p \rightarrow 0$ при $p \rightarrow \infty$, причем

$$
\varkappa(p) \ll p(\log \log p)^{2 / 3}(\log p)^{-1 / 9} .
$$

Заметим, что при достаточно больших $p$ из (1) вытекает асимптотика логарифма числа разностей в $\mathbf{Z}_{p}$.

\section{2. Определения и вспомогательные утверждения}

При доказательстве теоремы 3 нам понадобятся следующие понятия и вспомогательные утверждения.

Лемма 1 ([1]). Пусть $p$ - достаточно большое простое число, а $\mathscr{P}_{1}, \mathscr{P}_{2}-$ две арифметические прогрессии длинь $\lfloor p / 3\rfloor$ в $\mathbf{Z}_{p}$ с разностями, равными, соответственно, $d_{1}, d_{2}$ и при этом $d_{1} \neq \pm d_{2}$. Тогда

$$
\left|\mathscr{P}_{1} \cap \mathscr{P}_{2}\right| \leqslant \frac{p}{4}+4
$$

Пусть $A_{1}, A_{2}-$ непустые подмножества группы $\mathbf{Z}_{p}$. Обозначим через $S_{h}\left(A_{1}, A_{2}\right)$ множество элементов $x \in \mathbf{Z}_{p}$, для которых число представлений в виде $x=x_{1}+x_{2}$, $x_{1} \in A_{1}, x_{2} \in A_{2}$, не меньше $h$.

Лемма 2 ([2]). Пусть $p-$ простое число, $A_{1}, A_{2}-$ непустье подмножества группь $\mathbf{Z}_{p}$, и $h>0$ выбрано так, что

$$
(h p)^{1 / 2} \leqslant \min \left(\left|A_{1}\right|,\left|A_{2}\right|\right) .
$$

Тогда справедлива оценка

$$
\left|S_{h}\left(A_{1}, A_{2}\right)\right| \geqslant \min \left(p,\left|A_{1}\right|+\left|A_{2}\right|\right)-2(h p)^{1 / 2} .
$$

Пусть $L-$ натуральное число. Для каждого $y \in \mathbf{Z}_{p}$ определим разбиение $R_{y, L}$ группы $\mathbf{Z}_{p}$ на интервалы вида

$$
J_{i}^{y}=[i L+1+y,(i+1) L+y], \quad 0 \leqslant i \leqslant\lfloor p / L\rfloor-1 .
$$


Все интервалы $J_{i}^{y}$ из $R_{y, L}$ имеют длину $L$, а множество

$$
J_{y}=\mathbf{Z}_{p} \backslash \bigcup_{i} J_{i}^{y}
$$

имеет мощность $p-L\lfloor p / L\rfloor<L$. Множество $A \subseteq \mathbf{Z}_{p}$ назовем $L$-гранулированным, если для некоторого $d \in \mathbf{Z}_{p} \backslash\{0\}$ и разбиения $R_{y, L}$ группы $\mathbf{Z}_{p}$ множество $\{d a \mid a \in A\}$ является объединением нескольких интервалов $J_{i}^{y}$ разбиения $R_{y, L}$, отличных от $J_{y}$. Через $G_{L}\left(\mathbf{Z}_{p}\right)$ обозначим множество всех $L$-гранулированных подмножеств группы $\mathbf{Z}_{p}$.

Лемма 3 ([2]). Справедлива оценка

$$
\left|G_{L}\left(\mathbf{Z}_{p}\right)\right| \leqslant p 2^{p / L}
$$

Лемма 4 ([2]). Пусть $A \subseteq \mathbf{Z}_{p},|A|=\alpha p, u \varepsilon_{1}, \varepsilon_{2}, \varepsilon_{3}-$ положительные действительные числа, L-натуральное число, а $p$ - простое число, такое, что

$$
p>(4 L)^{256 \alpha^{2} \varepsilon_{1}^{-4} \varepsilon_{2}^{-2} \varepsilon_{3}^{-1}} .
$$

Тогда существует подмножество $A^{\prime} \subseteq \mathbf{Z}_{p}$ со следующими свойствами:

(i) $A^{\prime}$ является L-гранулированным;

(ii) $\left|A \backslash A^{\prime}\right| \leqslant \varepsilon_{1} p$;

(iii) множество $A-A$ содержит все элементы $x \in \mathbf{Z}_{p}$, для которых число представлений в виде $x=x_{1}-x_{2}, x_{1}, x_{2} \in A^{\prime}$, не меньше $\varepsilon_{2} p$, за исключением не более чем $\varepsilon_{3}$ р элементов.

\section{3. Нижняя оценка числа разностей в $\mathrm{Z}_{p}$}

Пусть

$$
L=\left\lfloor\frac{p}{6}\right\rfloor-1
$$

и

$$
\mathscr{P}=\{a+d, a+2 d, \ldots, a+(4 L+1) d\}
$$

- арифметическая прогрессия длины $4 L+1$ в $\mathbf{Z}_{p}$. Будем говорить, что подмножество $\mathscr{L} \subseteq \mathbf{Z}_{p}$ точно содержит $\mathscr{P}$, если $\mathscr{P} \subseteq \mathscr{X}$ и по крайней мере одно из $а$ и $a+(4 L+2) d$ не принадлежит $\mathscr{X}$. Положим

$$
\mathbf{S S}_{1,1}\left(\mathbf{Z}_{p}, A^{\prime}\right)=\left\{A \mid A \in \mathbf{S S}_{1,1}\left(\mathbf{Z}_{p}\right) \text { и } A \text { точно содержит } A^{\prime}\right\} \text {. }
$$

Лемма 5. Пусть $\mathscr{P} \subseteq \mathbf{Z}_{p}-$ произвольная арифметическая прогрессия длинь $4 L+1$. Тогда существует положительная константа с, такая, что

$$
\left|\mathbf{S S}_{1,1}\left(\mathbf{Z}_{p}, \mathscr{P}\right)\right| \geqslant c 2^{p / 3}
$$


Доказательство. Без ограничения общности можно считать, что

$$
\mathscr{P}=[-2 L, 2 L]
$$

Пусть $B \subseteq[-L, L] \backslash\{0\}$. Определим множество $A(B)$ равенством

$$
A(B)=(B \cup\{2 L+1\})-(B \cup\{2 L+1\}) .
$$

Нетрудно убедиться, что различные $B \subseteq[-L, L] \backslash\{0\}$ порождают различные множества $A(B)$, и ни одно из $-(2 L+1)$ и $2 L+1$ не принадлежит $A(B)$.

Множество $B \subseteq[-L, L] \backslash\{0\}$ определим следующим образом:

$$
B=B(C)=[-L,-L+15] \cup[-15,-1] \cup[1,15] \cup[L-15, L] \cup C,
$$

где элементы множества $C$ выбраны из $[-L+16,-16] \cup[16, L-16]$ случайно с вероятностью $1 / 2$, независимо друг от друга. Заметим, что

$$
[-2 L,-2 L+30] \cup[-30,30] \cup[2 L-30,2 L] \subseteq B-B .
$$

Будем говорить, что пары $\left(x_{1}, x_{2}\right)$ и $\left(y_{1}, y_{2}\right)$ не пересекаются, если

$$
\left\{x_{1}, x_{2}\right\} \cap\left\{y_{1}, y_{2}\right\}=\varnothing .
$$

Покажем, что для любого $x \in[31, L]$ выполняется неравенство

$$
\mathbf{P}(x \notin B-B) \leqslant\left(\frac{3}{4}\right)^{\lfloor x / 2\rfloor} .
$$

Положим

$$
Q_{L}(x)=\left\{\left(y_{1 i}, y_{2 i}\right) \mid x=y_{1 i}-y_{2 i}, y_{1 i}, y_{2 i} \in[-L, L] \backslash\{0\}, i=1, \ldots, m\right\},
$$

и

$$
D_{L}(x)=\left\{\left(z_{1 j}, z_{2 j}\right) \in Q_{L}(x) \mid\left\{z_{1 i}, z_{2 i}\right\} \cap\left\{z_{1 k}, z_{2 k}\right\}=\varnothing, i \neq k, j, i, k=1, \ldots, n\right\} .
$$

Тогда

$$
\begin{aligned}
\mathbf{P}(x \notin B-B) & \leqslant \mathbf{P}\left(\left(y_{11}-y_{21} \notin B-B\right) \& \ldots \&\left(y_{1 m}-y_{2 m} \notin B-B\right)\right) \\
& \leqslant \mathbf{P}\left(\left(z_{11}-z_{21} \notin B-B\right) \& \ldots \&\left(z_{1 n}-z_{2 n} \notin B-B\right)\right) \\
& =\mathbf{P}\left(\left(z_{11} \notin B \vee z_{21} \notin B\right) \& \ldots \&\left(z_{1 n} \notin B \vee z_{2 n} \notin B\right)\right) \\
& =\mathbf{P}\left(\left(z_{11} \notin B\right) \vee\left(z_{21} \notin B\right)\right) \cdots \mathbf{P}\left(\left(z_{1 n} \notin B\right) \vee\left(z_{2 n} \notin B\right)\right) \\
& =\mathbf{P}\left(\overline{\left(z_{11} \in B\right) \&\left(z_{21} \in B\right)}\right) \ldots \mathbf{P}\left(\overline{\left(z_{1 n} \in B\right) \&\left(z_{2 n} \in B\right)}\right) \\
& =\left(1-\mathbf{P}\left(\left(z_{11} \in B\right) \&\left(z_{21} \in B\right)\right)\right) \cdots\left(1-\mathbf{P}\left(\left(z_{1 n} \in B\right) \&\left(z_{2 n} \in B\right)\right)\right) .
\end{aligned}
$$

Отсюда и из того, что для любого $x \in[31, L]$ существует по крайней мере $\lfloor x / 2\rfloor$ непересекающихся пар, вытекает неравенство (3).

Аналогично с (3), для любого $x \in[31, L]$ выполняется неравенство

$$
\mathbf{P}(-x \notin B-B) \leqslant\left(\frac{3}{4}\right)^{\lfloor x / 2\rfloor} .
$$


Также нетрудно убедиться, что для любого $x \in[L+1,2 L-31]$ справедливы неравенства

$$
\mathbf{P}(x \notin B-B) \leqslant\left(\frac{3}{4}\right)^{\lfloor(2 L-x) / 2\rfloor}
$$

и

$$
\mathbf{P}(-x \notin B-B) \leqslant\left(\frac{3}{4}\right)^{\lfloor(2 L-x) / 2\rfloor} .
$$

Из вышесказанного следует, что

$$
\mathbf{P}(\mathscr{P} \nsubseteq B-B) \leqslant 4 \sum_{x \geqslant 31}\left(\frac{3}{4}\right)^{\lfloor x / 2\rfloor} .
$$

Действительно,

$$
\begin{aligned}
\mathbf{P}(\mathscr{P} \nsubseteq B-B) & =\mathbf{P}(-2 L \notin B-B) \vee \ldots \vee(2 L \notin B-B)) \leqslant \sum_{x=-2 L}^{2 L} \mathbf{P}(x \notin B-B) \\
& =\sum_{x=-2 L+31}^{-31} \mathbf{P}(x \notin B-B)+\sum_{x=31}^{2 L-31} \mathbf{P}(x \notin B-B) \\
& \leqslant 4 \sum_{x=31}^{L}\left(\frac{3}{4}\right)^{\lfloor x / 2\rfloor} \leqslant 4 \sum_{x \geqslant 31}\left(\frac{3}{4}\right)^{\lfloor x / 2\rfloor} .
\end{aligned}
$$

Заметим, что правая часть неравенства (5) не превосходит $1 / 2$. Отсюда следует, что существует по крайней мере $2^{2 L-63}$ подмножеств $B \subseteq[-L, L] \backslash\{0\}$, таких, что $\mathscr{P} \subseteq B-B$. Лемма доказана.

Пусть $\mathscr{P}_{1}, \mathscr{P}_{2} \subseteq \mathbf{Z}_{p}-$ две различные арифметические прогрессии длины $4 L+1$ с разностями, равными, соответственно, $d_{1}$ и $d_{2}$. Покажем, что количество множеств, которые точно содержат $\mathscr{P}_{1}$ и $\mathscr{P}_{2}$, есть $o\left(2^{p / 3}\right)$. Заметим, что при этом $d_{1} \neq \pm d_{2}$. В силу леммы 1 получаем, что

$$
\left|\overline{\mathscr{P}}_{1} \cap \overline{\mathscr{P}}_{2}\right| \leqslant \frac{p}{4}+20
$$

то есть

$$
\left|\mathscr{P}_{1} \cup \mathscr{P}_{2}\right| \geqslant \frac{3}{4} p-20
$$

Отсюда следует, что число множеств, которые точно содержат $\mathscr{P}_{1}$ и $\mathscr{P}_{2}$, есть $o\left(2^{p / 3}\right)$.

Итак, применяя лемму 5 для каждого из всевозможных $p(p-1) / 2$ вариантов арифметических прогрессий длины $4 L+1$, получаем нижнюю оценку.

Таким образом, нижняя оценка теоремы 3 доказана.

\section{4. Верхняя оценка числа разностей в $\mathbf{Z}_{p}$}

В этом разделе оценим сверху величину $\left|\mathbf{S S}_{1,1}\left(\mathbf{Z}_{p}\right)\right|$. Заметим, что число подмножеств группы $\mathbf{Z}_{p}$ мощности, не превышающей $p / 17$, есть $O\left(2^{p / 3}\right)$. 
Пусть $A \subseteq \mathbf{Z}_{p}$,

$$
|A|=\alpha p>\frac{p}{17}
$$

и $p$ - простое число, такое, что для натурального $L$ и положительных действительных чисел $\varepsilon_{1}, \varepsilon_{2}$ и $\varepsilon_{3}$ выполняется неравенство

$$
p>(4 L)^{256 \alpha^{2} \varepsilon_{1}^{-4} \varepsilon_{2}^{-2} \varepsilon_{3}^{-1}} .
$$

В силу леммы 4, существует подмножество $A^{\prime}$, обладающее свойствами (i)-(iii). Оценим величину $\left|\mathbf{S S}_{1,1}\left(\mathbf{Z}_{p}\right)\right|$ путем подсчета числа соответствующих пар $\left(A^{\prime}, A-A\right)$. Рассмотрим два случая: $\left|A^{\prime}\right| \geqslant p / 3$ и $\left|A^{\prime}\right|<p / 3$.

Пусть $\left|A^{\prime}\right| \geqslant p / 3$. В силу утверждения (iii) леммы 4 , множество $\overline{A-A}$ является подмножеством объединения множества $\overline{S_{\varepsilon_{2} p}\left(A^{\prime},-A^{\prime}\right)}$ и некоторого подмножества группы $\mathbf{Z}_{p}$ мощности, не превышающей $\varepsilon_{3} p$ и, следовательно,

$$
|\overline{A-A}| \leqslant\left|\overline{S_{\varepsilon_{2} p}\left(A^{\prime},-A^{\prime}\right)}\right|+\varepsilon_{3} p .
$$

В силу леммы 2 имеем

$$
\left|S_{\varepsilon_{2} p}\left(A^{\prime},-A^{\prime}\right)\right| \geqslant \min \left(p, 2\left|A^{\prime}\right|\right)-2\left(\varepsilon_{2} p^{2}\right)^{1 / 2} .
$$

При $\left|A^{\prime}\right| \geqslant p / 3$ получаем, что

$$
\begin{aligned}
\left|\overline{S_{\varepsilon_{2} p}\left(A^{\prime},-A^{\prime}\right)}\right| & =p-\left|S_{\varepsilon_{2} p}\left(A^{\prime},-A^{\prime}\right)\right| \\
& \leqslant \frac{p}{3}+2 \varepsilon_{2}{ }^{1 / 2} p .
\end{aligned}
$$

Отсюда и из (6) следует, что

$$
|\overline{A-A}| \leqslant \frac{p}{3}+2 \varepsilon_{2}^{1 / 2} p+\varepsilon_{3} p .
$$

Таким образом, учитывая, что множество $\overline{A-A}$ однозначно определяет множество $A-A$, получаем, что число способов выбора $A-A$ при заданном $A^{\prime}$ мощности, превышающей $p / 3$, не превосходит $2^{p / 3+\left(2 \varepsilon_{2}{ }^{1 / 2}+\varepsilon_{3}\right) p}$.

Пусть $\left|A^{\prime}\right|<p / 3$. В силу утверждения (ii) леммы 4,

$$
\left|A \backslash A^{\prime}\right| \leqslant \varepsilon_{1} p .
$$

Отсюда, учитывая, что каждое множество $A \subseteq \mathbf{Z}_{p}$ порождает ровно одно множество вида $A-A$, получаем, что число способов выбора $A-A$ при заданном $A^{\prime}$ мощности, не превышающей $p / 3$, не превосходит $2^{p / 3+\varepsilon_{1} p}$.

Из вышесказанного, с учетом леммы 3 и леммы 4 с параметрами

$$
\varepsilon_{1}=\varepsilon_{3}=\varepsilon, \quad \varepsilon_{2}=\varepsilon^{2}, \quad L=1+\left\lfloor\frac{1}{\varepsilon}\right\rfloor,
$$

получаем, что

$$
\left|\mathbf{S S}_{1,1}\left(\mathbf{Z}_{p}\right)\right| \leqslant 2^{p / 3+C \varepsilon p} .
$$

Несложно убедиться, что можно взять

$$
\varepsilon=O\left((\log \log p)^{2 / 3}(\log p)^{-1 / 9}\right) .
$$

Таким образом, верхняя оценка, а значит, и теорема 3 доказаны. 


\section{Список литературы}

1. Green B., Ruzsa I., Counting sumsets and sum-free sets modulo a prime. Studia Sci. Math. Hungarica (2004) 41, 285-293.

2. Sargsyan V., Counting $(k, l)$-sumsets in groups of a prime order. Acta Universitatis Sapientiae, Informatica (2012) 4, №1, 33-47.

Статья поступила 01.02.2013. 